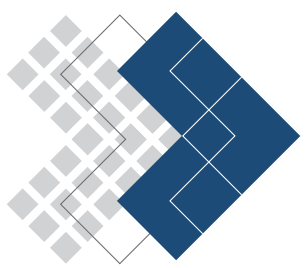 \\ VETOR
}

\section{Avanços Recentes na Aplicação de um Método Numérico Espectronodal para Solução de Problemas Unidimensionais de Blindagem de Nêutrons}

\section{Recent Advances on the Application of a Spectral Nodal Numerical Method for the Solution of One-Dimensional Neutron Shielding Problems}

\author{
Rafael Barbosa Libotte ${ }^{\dagger}$, Hermes Alves Filho, Fernando Carvalho da Silva \\ ${ }^{1}$ Instituto Politécnico, Universidade do Estado do Rio de Janeiro, Nova Friburgo, Brasil \\ ${ }^{\dagger}$ Autor correspondente: rafaellibotte@hotmail.com
}

\section{Resumo}

Neste trabalho, é mostrada a aplicação de um método da classe dos espectronodais (malha grossa) na solução de problemas de blindagem de nêutrons em geometria unidimensional nas formulações de ordenadas discretas e multigrupo de energia. Este método, denominado Modified Spectral Deterministic (MSD), representou uma modificação na estrutura de obtenção dos fluxos angulares de nêutrons emergentes no processo de varredura que foi utilizado no Método Espectral Deterministico (do inglês Spectral Deterministic Method, SDM). São apresentados os resultados numéricos para 3 problemas-modelo, com diferentes dimensões, número de grupos de energia, grau de anisotropia no fenômeno de espalhamento e tipos de condição de contorno, usando um aplicativo computacional desenvolvido na linguagem de programação $\mathrm{C}++$.

\section{Palavras-chave}

Teoria de transporte de nêutrons • Blindagem de nêutrons • Métodos Espectronodais • Modelagem computacional determinística • Neutrônica computacional

\begin{abstract}
In this work, it is presented the application of a spectral nodal method (coarse-mesh) in the solution of one-dimensional neutron shielding problems, in the discrete ordinates and energy multigroup formulations. This method, named Modified Spectral Deterministic (MSD), represents a modification of the calculation structure of the neutron angular flux in the sweeping process used in the Spectral Deterministic Method (SDM). The numerical results for 3 modelproblems are shown, with different dimensions, number of energy groups and types of boundary conditions in using a computational application developed in the programming language $\mathrm{C}++$.
\end{abstract}

\section{Keywords}

Neutrons transport theory $\cdot$ Neutron shielding - Spectral nodal methods - Deterministic computational modelling • Computational neutronics

\section{Introdução}

A fim de analisar o comportamento da migração de nêutrons em um meio hospedeiro, é necessário realizar o estudo do transporte destas partículas neste domínio. Uma equação que pode ser utilizada para modelar matematicamente 
esse fenômeno físico é a equação linear de Boltzmann, que representa neste caso um balanço entre a quantidade de nêutrons produzidos e removidos no interior de um domínio de interesse [1].

Com o passar do tempo, diferentes abordagens numéricas foram usadas para resolver o problema de transporte de nêutrons, usando esta formulação determinística. Métodos de malha fina foram desenvolvidos para aplicação nestes problemas, como o tradicional método Diamond Difference (DD) [2], usado como referência em resultados numéricos até hoje. A utilização de métodos de malha fina pode ser tornar computacionalmente muito custosa, quando uma maior precisão é necessária em casos mais complexos. Portanto, o desenvolvimento de métodos de malha grossa [3, 4, 5, 6] tornaram-se importantes para a solução de problemas de neutrônica em diversos campos das ciências e engenharias.

Dentro da classe dos métodos de malha grossa (nodais), temos os métodos espectronodais [4, 5, 7, 3, 8, 6]. Neste trabalho, apresentamos resultados numéricos recentes do método espectronodal Modified Spectral Deterministic (MSD) [4] para cálculos de blindagem de nêutrons usando a equação multigrupo de transporte em geometria unidimensional na formulação das ordenadas discretas $\left(S_{N}\right)$.

Parte da construção do algoritmo numérico de solução do método MSD, envolve um processo iterativo que é baseado em operações matriciais, iguais aos que foram desenvolvidos no método Spectral Derterministic Method [5]. Porém, o cálculo dos fluxos angulares emergentes de nêutrons nos cantos nodos são feito a partir de duas equações, usando as equações de balanço espacial $S_{N}$ [4]. Tentou-se, com esse procedimento, minimizar a realização de algumas operações matriciais, que podem aumentar o tempo de execução dos algoritmos numéricos, ou mesmo, tornar instáveis esses algoritmos na modelagem computacional de alguns problemas-modelos testados. São apresentados os resultados numéricos para 3 problemas-modelo típicos para testar a precisão do método espectronodal MSD na solução de problemas de blindagem de nêutrons.

A seguir são apresentadas as seções que compõem este trabalho. Na Seção 2, é mostrada a modelagem matemática do fenômeno de transporte de nêutrons em um meio hospedeiro não-multiplicativo (fonte-fixa) em geometria unidimensional, na formulação das ordenadas discretas, usando a teoria de multigrupos de energia. Na Seção 3, é apresentado o desenvolvimento das equações que compõem o método MSD. Na Seção 4, são mostrados os resultados numéricos de 2 problemas-modelo típicos de blindagem de nêutrons. Finalmente, na Seção 5, são mostradas as conclusões acerca deste trabalho, bem como as propostas para trabalhos futuros.

\section{Modelo matemático}

O fenômeno de transporte de nêutrons em um meio material pode ser modelado matematicamente segundo a equação linear de Boltzmann, originalmente aplicada no estudo da teoria da cinética de gases [1]. Esta equação é centrada no balanço dos eventos que provocam a produção e remoção das partículas neutras, considerando algumas hipóteses a priori [2], sendo estas:

- Os nêutrons são considerados como partículas pontuais: a distância média de migração de um nêutron entre colisões é muito maior que sua dimensão.

- A trajetória de migração de uma partícula entre duas colisões é considerada uma reta: visto que nêutrons não possuem cargas, a sua direção de migração não é alterada por campos eletro-magnéticos, e forças gravitacionais não são fortes o suficiente para mudar a trajetória de uma partícula. Portanto, é razoável considerar que o nêutron migra em linha reta entre suas colisões.

- Interações partícula-partícula são desconsideradas: a densidade de nêutrons é muito menor que a densidade das partículas constituintes do meio material. Portanto, é viável desconsiderar as colisões entre nêutrons.

- Colisões são consideradas instantâneas: após a colisão de um nêutron com um núcleo do meio material, as partículas emergentes são emitidas instantaneamente.

- Os materiais são isotrópicos: na equação linear de Boltzmann, efeitos que dependem da orientação das partículas não são considerados.

- As propriedades nucleares e os parâmetros físico-materiais são considerados independentes do tempo.

- Apenas o valor médio da densidade de distribuição de partículas é considerado: flutuações estatísticas dentro do elemento são desprezadas, uma vez que elementos de velocidade e espaço são admitidos como suficientemente grandes.

Na sua forma geral, a equação de transporte de nêutrons possui 7 variáveis independentes: sendo três espaciais, que podem ser discretizadas seguindo métodos de malha fina, e.g. Diamond Difference (DD) [2], ou de malha grossa, como os métodos espectronodais, e.g. Método Espectral Determinístico (SDM) [5], Matriz Resposta (RM) [3] ou 
Spectral Green's Function (SGF) [6]. A variável angular pode ser tratada segundo a formulação das ordenadas discretas [2], e a variável energia pelo método multigrupo [9]. Neste trabalho, foram abordados só casos independentes do tempo (regime permanente).

A equação que modela o problema de transporte de nêutrons, em um meio material não-multiplicativo, domínio unidimensional de comprimento $H$, com grau arbitrário de anisotropia para o fenômeno de espalhamento, formulação das ordenadas discretas, usando a teoria de multigrupos de energia é dada pela expressão

$$
\begin{aligned}
\mu_{m} \frac{d}{d x} \psi_{m, g}(x)+\sigma_{T, g}(x) \psi_{m, g}(x)=\frac{1}{2} \sum_{g^{\prime}=1}^{G}\left[\sum_{l=0}^{L}(2 l+1) \sigma_{S l}^{g^{\prime} \rightarrow g}(x) P_{l}\left(\mu_{m}\right) \sum_{n=1}^{N} P_{l}\left(\mu_{n}\right) \psi_{n, g}(x) \omega_{n}\right] \\
+Q_{g}(x), m=1: N, g=1: G, x \in[0, H]
\end{aligned}
$$

onde $\psi_{m, g}(x)$ representa o fluxo angular de nêutrons na direção discreta $m$ no grupo de energia $g$. Os valores $\mu_{m}$ representam as ordenadas discretas e são as raízes dos polinômios de Legendre de grau $N$. Os $\omega_{m}$ representam os pesos da quadratura de Gauss-Legendre de ordem $N$. As variáveis $\sigma_{T, g}$ e $\sigma_{S l}^{g^{\prime} \rightarrow g}$ representam as seções de choque macroscópicas total do grupo $g$ e a de espalhamento de grau $l$ do grupo $g^{\prime}$ para o $g$. Os $P_{l}$ são os polinômios de Legendre de grau $l$ e $Q_{g}$ é uma fonte externa isotrópica de nêutrons.

Este é um problema no qual os fluxos angulares de nêutrons incidentes nos contornos externos do domínio espacial são conhecidos, e podem atender a algumas condições de contorno:

- Condição de contorno do tipo prescrita: neste tipo, os fluxos angulares de nêutrons incidentes nas extremidades do domínio possuem valores conhecidos. Esta condição é dada por

$$
\psi_{m, g}(x)=\left\{\begin{array}{l}
b_{m, g}, \text { se } x=0 \text { e } \mu_{m}>0, g=1: G \\
c_{m, g}, \text { se } x=H \text { e } \mu_{m}<0, g=1: G,
\end{array}\right.
$$

- Condição de contorno do tipo vácuo: é um caso particular da prescrita, define que os fluxos angulares incidentes nos contornos são nulos, na forma

$$
\psi_{m, g}(x)=\left\{\begin{array}{l}
0, \text { se } x=0 \text { e } \mu_{m}>0, g=1: G \\
0, \text { se } x=H \text { e } \mu_{m}<0, g=1: G .
\end{array}\right.
$$

- Condição de contorno do tipo reflexiva: aqui, os fluxos angulares de nêutrons incidentes nos contornos esquerdo $(x=0)$ e direito $(x=H)$ assumem os mesmos valores dos fluxos angulares de nêutrons emergentes em ângulos complementares. Matematicamente, esta condição é escrita como

$$
\left\{\begin{array}{l}
\psi_{m, g}(0)=\psi_{m+N / 2, g}(0), \quad m=1: N / 2 \\
\psi_{m+N / 2, g}(H)=\psi_{m, g}(H), \quad m=1: N / 2
\end{array}\right.
$$

\subsection{Equação intranodal de transporte de nêutrons $S_{N}$}

No processo de discretização espacial, o domínio de comprimento $H$ é dividido em $J$ nodos $\Gamma_{j}$, com espessura $h_{j}$, admitindo que os parâmetros físico-materiais, bem como a fonte de espalhamento, sejam uniformes no interior do nodo $\Gamma_{j}$. Na Fig. 11 é mostrada a representação esquemática desta técnica.

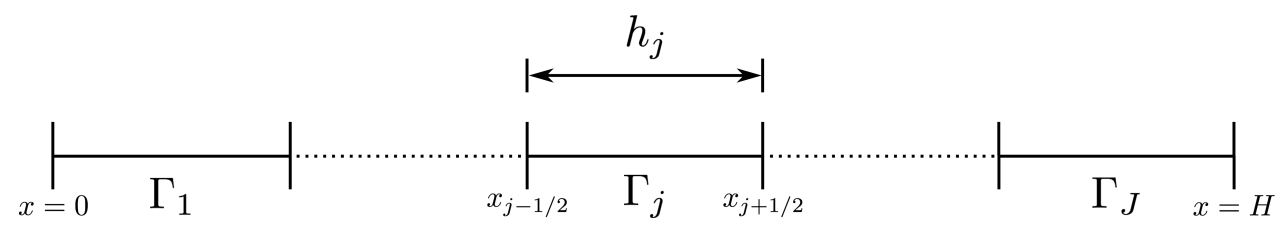

Figura 1: Discretização da variável espacial.

Ao aplicar a Eq. (1) no interior de um nodo arbitrário $\Gamma_{j}$, é obtida a equação intranodal de transporte de nêutrons $S_{N}$, no formato 


$$
\begin{aligned}
& \mu_{m} \frac{d}{d x} \psi_{m, g}(x)+\sigma_{T, g, j} \psi_{m, g}(x)=\frac{1}{2} \sum_{g^{\prime}=1}^{G}\left[\sum_{l=0}^{L}(2 l+1) \sigma_{S l, j}^{g^{\prime} \rightarrow g} P_{l}\left(\mu_{m}\right) \sum_{n=1}^{N} P_{l}\left(\mu_{n}\right) \psi_{n, g}(x) \omega_{n}\right]+Q_{g, j} \\
& m=1: N, g=1: G, x_{j-1 / 2} \leq x \leq x_{j+1 / 2} .
\end{aligned}
$$

\section{Modified Spectral Deterministic (MSD)}

Nesta seção, são mostradas as equações que compõem o método Modified SpectralDeterministic(MSD), para o cálculo dos fluxos angulares de nêutrons emergentes nas interfaces nodais. Este método é uma modificação do Método Espectral Determinístico (SDM) [5]. Ambas as metodologias nodais podem ser usadas na solução de problemas de blindagem de nêutrons. O MSD usa a solução analítica geral intranodal da equação de transporte de nêutrons $S_{N}$, similar ao SDM, e um processo iterativo que usa a equação de balanço espacial $S_{N}$ para o cálculo dos fluxos angulares de nêutrons emergentes nas interfaces nodais.

\subsection{Solução geral analítica local da equação de transporte $S_{N}$}

A solução analítica geral intranodal da Eq.(5) é composta por duas componentes, uma homogênea e uma particular, descrita como

$$
\psi_{m, g}(x)=\psi_{m, g}^{h}(x)+\psi_{m, g}^{p}, m=1: N, g=1: G .
$$

A fim de obter a solução particular $\left(\psi_{m, g}^{p}\right)$, é solucionado um sistema de equações [5], dado por

$$
\sum_{g^{\prime}=1}^{G} \sum_{n=1}^{N}\left(\sigma_{T, g, j} \delta_{m, n} \delta_{g^{\prime}, g}-\frac{1}{2} \sum_{l=0}^{L}(2 l+1) P_{l}\left(\mu_{m}\right) \sigma_{S l, j}^{g^{\prime} \rightarrow g} P_{l}\left(\mu_{n}\right) \omega_{n}\right) \psi_{n, g^{\prime}}^{p}=Q_{g, j}, j=1: J, m=1: N, g=1: G
$$

onde $\delta$ representa o Delta de Kroenecker. Para calcular as componentes homogêneas da solução $\left(\psi_{m, g}^{h}(x)\right)$, deve ser considerada a expressão [10, 11, 12]

$$
\psi_{m, g}^{h}(x)=a_{m, g}(\vartheta) \exp \left(\frac{-(x-\lambda)}{\vartheta}\right)
$$

onde $\lambda$ representa um parâmetro com propriedades computacionais, evitando problemas de overflow resultantes do expoente visto na Eq. (8). Este parâmetro é definido como

$$
\lambda=\left\{\begin{array}{l}
x_{j-1 / 2}, \text { se } \vartheta>0 \\
x_{j+1 / 2}, \text { se } \vartheta<0
\end{array}\right.
$$

Ao substituir a Eq. (8) na parte homogênea da Eq. (5), é obtido um problema de autovalor na forma

$$
\sum_{g^{\prime}=1}^{G} \sum_{n=1}^{N}\left[\frac{\delta_{m n} \delta_{g^{\prime} g} \sigma_{T, g, j}}{\mu_{m}}-\frac{\omega_{n}}{2 \mu_{m}} \sum_{l=0}^{N-1}(2 l+1) \sigma_{S l, j}^{g^{\prime} \rightarrow g} P_{l}\left(\mu_{m}\right) P_{l}\left(\mu_{n}\right)\right] a_{n, g^{\prime}}(\vartheta)=\frac{1}{\vartheta} a_{m, g}(\vartheta) .
$$

Na solução da Eq. (10), são obtidos $N G$ autovalores reais e simétricos $\left(\vartheta_{l}\right)$, e $N G$ autovetores $\left(a_{m}(\vartheta)\right)$ associados aos autovalores. Assim, a solução analítica geral intranodal da equação de transporte de nêutrons $S_{N}$ em um nodo arbitrário $\Gamma_{j}$, assume o forma

$$
\psi_{m, g}(x)=\sum_{l=1}^{N G} \alpha_{l} a_{m, g}\left(\vartheta_{l}\right) \exp \left(\frac{-(x-\lambda)}{\vartheta_{l}}\right)+\psi_{m, g}^{p}
$$

onde $\alpha_{l}$ representa um conjunto de $N G$ variáveis arbitrárias a serem determinadas [5]. 


\subsection{Equações de balanço espacial $S_{N}$}

Dando continuidade na montagem das equações constitutivas do MSD, é aplicado agora o operador média, descrito como

$$
\frac{1}{h_{j}} \int_{x_{j-1 / 2}}^{x_{j+1 / 2}}(\cdot) \mathrm{d} x
$$

na Eq. (5), resultando na equação de balanço espacial $S_{N}$, descrita como

$$
\begin{array}{r}
\frac{\mu_{m}}{h_{j}}\left(\psi_{m, g, j+1 / 2}-\psi_{m, g, j-1 / 2}\right)+\sigma_{T, g, j} \bar{\psi}_{m, g, j}=\frac{1}{2} \sum_{g^{\prime}=1}^{G}\left[\sum_{l=0}^{L}(2 l+1) \sigma_{S l, j}^{g^{\prime} \rightarrow g} P_{l}\left(\mu_{m}\right) \sum_{n=1}^{N} P_{l}\left(\mu_{n}\right) \bar{\psi}_{n, g^{\prime}, j} \omega_{n}\right]+Q_{g, j}, \\
m=1: N, g=1: G .
\end{array}
$$

O fluxo angular médio de nêutrons é definido por

$$
\bar{\psi}_{m, g, j} \equiv \frac{1}{h_{j}} \int_{x_{j-1 / 2}}^{x_{j+1 / 2}} \psi_{m, g}(x) \mathrm{d} x, m=1: N, g=1: G, j=1: J .
$$

Ao substituir o valor do fluxo angular de nêutrons $\psi_{m, g}(x)$ (Eq.(11)) na Eq. (14), é a obtida a expressão para os fluxos angulares médios de nêutrons, dada por

$$
\bar{\psi}_{m, g, j}=\frac{1}{h_{j}} \sum_{l=1}^{N G} \alpha_{l} a_{m, g}\left(\vartheta_{l}\right)\left|\vartheta_{l}\right|\left(1-e^{-h_{j} /\left|\vartheta_{l}\right|}\right)+\psi_{m, g}^{p}
$$

Após a obtenção destes valores, podem ser construídas as equações para o cálculo dos fluxos angulares de nêutrons emergentes nas interfaces dos nodos $\Gamma_{j}$ em função dos fluxos incidentes e fontes de nêutrons no interior do nodo arbitrário $\Gamma_{j}$. Na Fig. 2 é mostrada uma representação dos fluxos angulares de nêutrons incidentes e emergentes em um nodo arbitrário $\Gamma_{j}$, com a presença de uma fonte-fixa isotrópica $Q_{g, j}$.

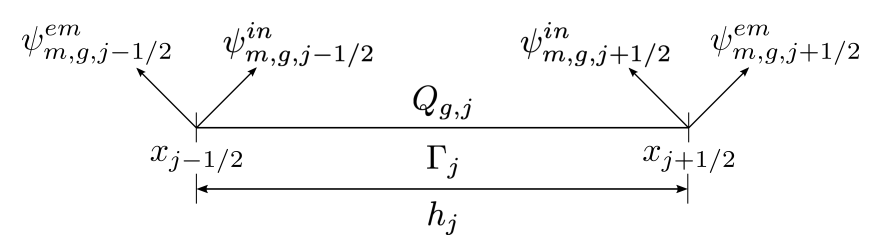

Figura 2: Representação dos fluxos angulares de nêutrons em um nodo arbitrário $\Gamma_{j}$. obtemos

O cálculo dos fluxos angulares de nêutrons emergentes no nodo $\Gamma_{j}$ é realizado usando a Eq. (13). Para $\mu_{m}>0$,

$$
\begin{array}{r}
\psi_{m, g, j+1 / 2}=\frac{h j}{\mu_{m}}\left(-\sigma_{T, g, j} \bar{\psi}_{m, g, j}+\frac{1}{2} \sum_{g^{\prime}=1}^{G}\left[\sum_{l=0}^{L}(2 l+1) \sigma_{S l, j}^{g^{\prime} \rightarrow g} P_{l}\left(\mu_{m}\right) \sum_{n=1}^{N} P_{l}\left(\mu_{n}\right) \bar{\psi}_{n, g^{\prime}, j} \omega_{n}\right]+Q_{g, j}\right)+\psi_{m, g, j-1 / 2}, \\
m=1: N / 2, g=1: G .
\end{array}
$$

para $\mu_{m}<0$, é obtida a equação

$$
\begin{array}{r}
\psi_{m, g, j-1 / 2}=\frac{h j}{\left|\mu_{m}\right|}\left(-\sigma_{T, g, j} \bar{\psi}_{m, g, j}+\frac{1}{2} \sum_{g^{\prime}=1}^{G}\left[\sum_{l=0}^{L}(2 l+1) \sigma_{S l, j}^{g^{\prime} \rightarrow g} P_{l}\left(\mu_{m}\right) \sum_{n=1}^{N} P_{l}\left(\mu_{n}\right) \bar{\psi}_{n, g^{\prime}, j} \omega_{n}\right]+Q_{g, j}\right) \\
m=N / 2+1: N, g=1: G .
\end{array}
$$




\subsection{Varredura da grade espacial}

De posse das Eqs. (16 17), o algoritmo do processo iterativo pode ser construido. Partindo de estimativas iniciais para os fluxos angulares de nêutrons e das condições de contorno, é possível calcular os fluxos angulares de nêutrons emergentes nas interfaces nodais iterativamente. Uma grade com 3 regiões e ordem de quadratura $N=4$ é mostrada na Fig. 3. a fim de exemplificar o processo de varredura.

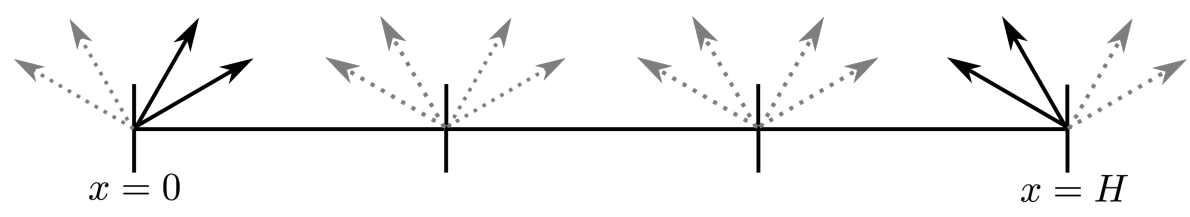

Figura 3: Representação das estimativas iniciais dos fluxos angulares de nêutrons em um nodo arbitrário $\Gamma_{j}$.

Na Fig. 3. as condições de contorno são representadas pelas setas sólidas pretas, as estimativas iniciais dos fluxos angulares de nêutrons pelas setas pontilhadas cinza.

O processo de varredura é feito percorrendo todos os nodos da grade espacial, da esquerda $(x=0)$ para a direita $(x=H)$. O primeiro passo é usar as condições de contorno à esquerda e os fluxos angulares de nêutrons incidentes estimados no primeiro nodo e calcular um conjunto de parâmetros $\alpha$, usando a Eq. (11) [5]. Agora, os fluxos médios de nêutrons neste nodo são calculados usando a Eq. (15). A partir destes valores, podemos estimar os seguintes termos:

$$
S_{S, m, g, j}=\frac{1}{2} \sum_{g^{\prime}=1}^{G}\left[\sum_{l=0}^{L}(2 l+1) \sigma_{S l, j}^{g^{\prime} \rightarrow g} P_{l}\left(\mu_{m}\right) \sum_{n=1}^{N} P_{l}\left(\mu_{n}\right) \bar{\psi}_{n, g^{\prime}, j} \omega_{n}\right]
$$

e

$$
T_{m, g, j}=-\sigma_{T, g, j} \bar{\psi}_{m, h, j}
$$

Neste ponto, os fluxos angulares de nêutrons emergentes do primeiro nodo podem ser calculados, à direita com a Eq. (16) e à esquerda de acordo com a Eq. (17), resultando no esquema mostrado na Fig. 4

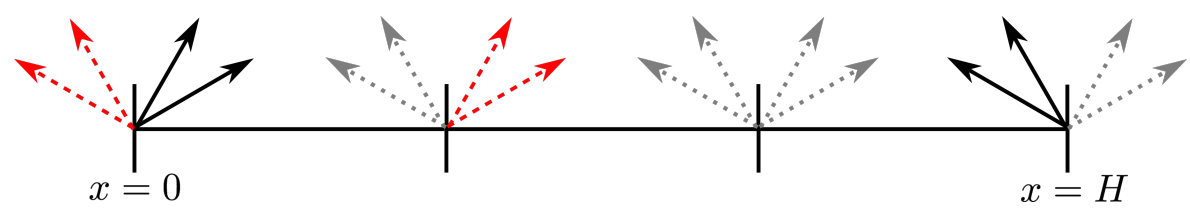

Figura 4: Representação do cálculos dos fluxos angulares de nêutrons emergentes no primeiro passo da varredura.

Onde as setas tracejadas vermelhas representam os fluxos angulares de nêutrons emergentes atualizados no primeiro nodo.

Nota-se na Fig. 4 que o fluxo angular de nêutrons emergentes á direita do primeiro nodo coincide com o fluxo angular de nêutrons incidente à esquerda do segundo nodo. A partir desta estimativa, o mesmo processo pode ser realizado para que os fluxos angulares de nêutrons emergentes do segundo nodo possam ser calculados. Este processo é repetido até que toda a grade espacial seja percorrida, e todos os fluxos angulares de nêutrons emergentes de cada um dos nodos do domínio seja atualizado.

Neste ponto, uma iteração é finalizada, e o critério de parada pode ser avaliado, segundo a expressão

$$
\max _{g, j}\left|\frac{\phi_{g, j-1 / 2}^{(k)}-\phi_{g, j-1 / 2}^{(k-1)}}{\phi_{g, j-1 / 2}^{(k-1)}}\right|<\xi, j=1: J+1, g=1: G,
$$

onde $\xi$ representa um parâmetro de precisão preestabelecido, $k$ representa o número da atual iteração e $\phi$ os fluxos escalares de nêutrons nas interfaces nodais, calculados com a equação

$$
\phi_{g, j-1 / 2}^{(k)}=\frac{1}{2} \sum_{n=1}^{N} \psi_{n, g, j-1 / 2}^{(k)} \omega_{n}, j=1: J+1 .
$$




\section{Resultados numéricos}

Nesta seção, são apresentados os resultados numéricos do cálculo dos fluxos escalares de nêutrons nas interfaces nodais em 3 problemas-modelo. Os resultados numéricos gerados usando o método MSD são comparados com alguns métodos encontrados na literatura.

\subsection{Problema-modelo 1}

O primeiro problema-modelo deste trabalho é composto por um domínio de $100 \mathrm{~cm}$ de espessura, com parâmetros físico-materiais uniformes, como mostrado na Fig. 5. Neste modelo, são considerados 2 grupos de energia. As condições de contorno são do tipo prescrita no contorno esquerdo, e do tipo vácuo no direito.

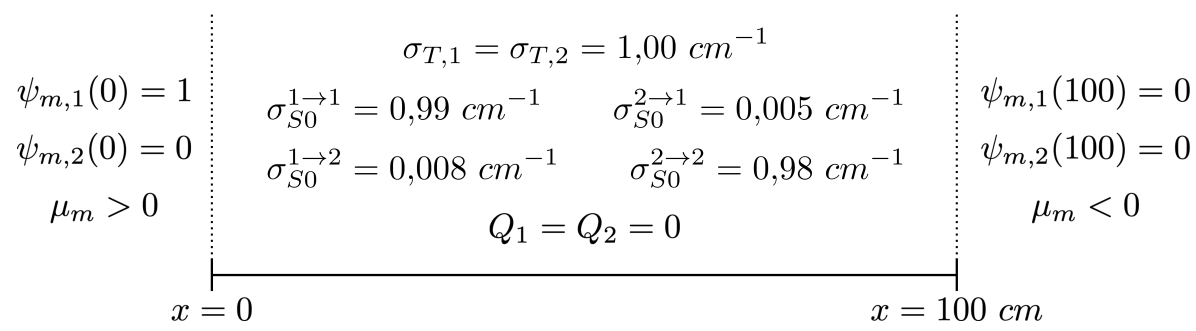

Figura 5: Problema-modelo 1.

A execução dos métodos de malha grossa MSD e SDM foram feitas com um nodo na região que compõe o domínio. Para o método DD, foi usada uma grade espacial com 10000 nodos. Na solução de todos os problemas-modelo por métodos de malha fina, a grade espacial utilizada foi refinada até que não houvessem mais alterações nos valores dos fluxos escalares de nêutrons, considerando o números de algarismos significativos coincidentes com a precisão utilizada $(\xi)$. Para todos os métodos foi usado o parâmetro $\xi=10^{-6}$, no critério de parada do processo iterativo. Os resultados numéricos para os fluxos escalares de nêutrons $\left(\mathrm{cm}^{-2} \mathrm{~s}^{-1}\right)$ nos contornos do domínio espacial são mostrados na Tabela 1 para as ordens de quadratura $N=4$ e $N=16$.

Tabela 1: Fluxos escalares $\left(\mathrm{cm}^{-2} \mathrm{~s}^{-1}\right)$ do problema-modelo 1.

\begin{tabular}{lcccccc}
\hline & & \multicolumn{2}{c}{$N=4$} & \multicolumn{2}{c}{$N=16$} \\
\cline { 3 - 4 } \cline { 6 - 6 } Método & Grupo & $x=0 \mathrm{~cm}$ & $x=100 \mathrm{~cm}$ & & $x=0 \mathrm{~cm}$ & $x=100 \mathrm{~cm}$ \\
\hline \multirow{2}{*}{ DD } & 1 & $9,12675 \times 10^{-1}$ & $6,27623 \times 10^{-8}$ & & $9,12675 \times 10^{-1}$ & $6,24863 \times 10^{-8}$ \\
& 2 & $2,72638 \times 10^{-2}$ & $3,84382 \times 10^{-8}$ & & $2,72638 \times 10^{-2}$ & $3,82692 \times 10^{-8}$ \\
\hline \multirow{2}{*}{ MSD } & 1 & $9,12675 \times 10^{-1}$ & $6,27693 \times 10^{-8}$ & & $9,12675 \times 10^{-1}$ & $6,24932 \times 10^{-8}$ \\
& 2 & $2,72639 \times 10^{-2}$ & $3,84425 \times 10^{-8}$ & & $2,72639 \times 10^{-2}$ & $3,82734 \times 10^{-8}$ \\
\hline \multirow{2}{*}{ SDM } & 1 & $9,12675 \times 10^{-1}$ & $6,27693 \times 10^{-8}$ & & $9,12675 \times 10^{-1}$ & $6,24932 \times 10^{-8}$ \\
& 2 & $2,72639 \times 10^{-2}$ & $3,84425 \times 10^{-8}$ & & $2,72639 \times 10^{-2}$ & $3,82734 \times 10^{-8}$ \\
\hline
\end{tabular}

Os resultados numéricos mostrados na Tabela 1 indicam que o método MSD obteve uma boa precisão na obtenção dos fluxos escalares, quando comparado com os métodos DD (malha fina) e SDM (malha grossa).

\subsection{Problema-modelo 2}

No segundo problema-modelo, apresentamos um caso com um domínio de $100 \mathrm{~cm}$ de espessura, e 3 zonas materiais, considerando 2 grupos de energia. Na Fig. 6 são mostrados os parâmetros físico-materiais, bem como as espessuras e a distribuição da fonte de externa de nêutrons de cada região. Este problema possui condições de contorno do tipo reflexiva no contorno esquerdo e do tipo vácuo no direito. 


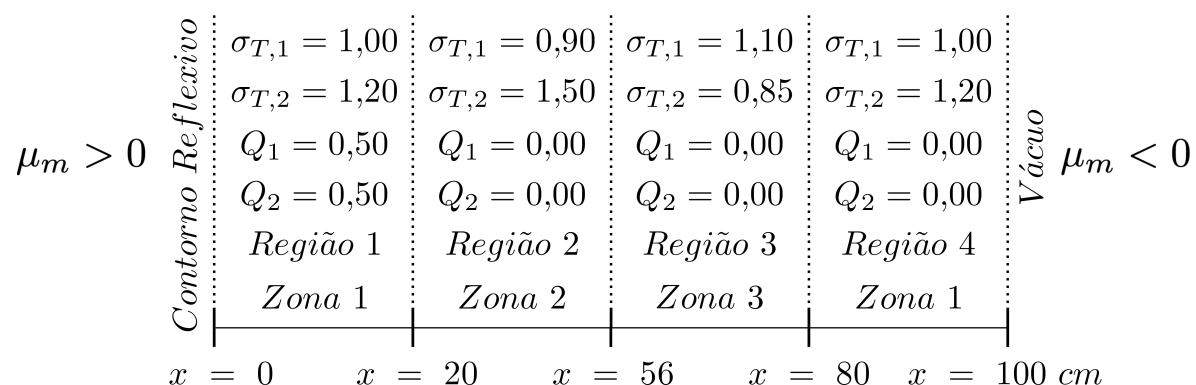

Figura 6: Problema-modelo 2

A Tabela 2 mostra as seções de choque de espalhamento das 3 zonas materiais constituintes do domínio deste problema.

Tabela 2: Seções de choque macroscópicas de espalhamento $\left(\mathrm{cm}^{-1}\right)$ do problema-modelo 2.

\begin{tabular}{ccccccccc}
\hline & \multicolumn{2}{c}{ Zona 1} & & \multicolumn{2}{c}{ Zona 2} & & \multicolumn{2}{c}{ Zona 3 } \\
\cline { 2 - 3 } Método & $g=1$ & $g=2$ & & $g=1$ & $g=2$ & & $g=1$ & $g=2$ \\
\hline$\sigma_{S 0}^{1 \rightarrow g}$ & 0,90 & 0,20 & & 0,75 & 0,30 & & 0,95 & 0,60 \\
$\sigma_{S 0}^{2 \rightarrow g}$ & 0,05 & 0,80 & & 0,10 & 0,99 & & 0,0 & 0,20 \\
\hline
\end{tabular}

Os fluxos escalares de nêutrons deste problema foram calculados usando um parâmetro de tolerância $\xi=10^{-6}$. Os métodos de malha grossa MSD e SDM foram executados com um nodo por região. No método DD foi utilizada uma malha com 1000 nodos na primeira e quarta região, 1800 na segunda região e 1200 na terceira. Os resultados numéricos para os fluxos escalares de nêutrons $\left(\mathrm{cm}^{-2} \mathrm{~s}^{-1}\right)$ deste problema são mostrados para as ordens de quadratura $N=32$ e $N=128$ na Tabela 3

Tabela 3: Fluxos escalares de nêutrons $\left(\mathrm{cm}^{-2} \mathrm{~s}^{-1}\right)$ - problema-modelo 2.

\begin{tabular}{lcccccc}
\hline \multirow{2}{*}{ Método } & Grupo & $x=0 \mathrm{~cm}$ & $x=20 \mathrm{~cm}$ & $x=56 \mathrm{~cm}$ & $x=80 \mathrm{~cm}$ & $x=100 \mathrm{~cm}$ \\
\cline { 2 - 7 } DD & 1 & 7,49903 & 3,51557 & $1,86864 \times 10^{-7}$ & $2,70421 \times 10^{-14}$ & $1,58405 \times 10^{-18}$ \\
& 2 & 4,99943 & 2,55794 & $1,36673 \times 10^{-7}$ & $3,42809 \times 10^{-14}$ & $8,30350 \times 10^{-19}$ \\
\hline \multirow{2}{*}{ MSD } & 1 & 7,49903 & 3,51557 & $1,87413 \times 10^{-7}$ & $2,72811 \times 10^{-14}$ & $1,60046 \times 10^{-18}$ \\
& 2 & 4,99943 & 2,55794 & $1,37074 \times 10^{-7}$ & $3,45835 \times 10^{-14}$ & $8,38952 \times 10^{-19}$ \\
\hline \multirow{2}{*}{ SDM } & 1 & 7,49902 & 3,51557 & $1,87413 \times 10^{-7}$ & $2,72811 \times 10^{-14}$ & $1,60046 \times 10^{-18}$ \\
& 2 & 4,99943 & 2,55794 & $1,37074 \times 10^{-7}$ & $3,45835 \times 10^{-14}$ & $8,38952 \times 10^{-19}$ \\
\hline \multirow{2}{*}{ DD } & & & & $N=128$ & \\
& 2 & 4,99943 & 2,55791 & $1,37069 \times 10^{-7}$ & $3,45715 \times 10^{-14}$ & $8,38569 \times 10^{-19}$ \\
\hline \multirow{2}{*}{ MSD } & 1 & 7,49903 & 3,51559 & $1,87411 \times 10^{-7}$ & $2,72813 \times 10^{-14}$ & $1,60032 \times 10^{-18}$ \\
& 2 & 4,99943 & 2,55791 & $1,37085 \times 10^{-7}$ & $3,45837 \times 10^{-14}$ & $8,38927 \times 10^{-19}$ \\
\hline \multirow{2}{*}{ SDM } & 1 & 7,49903 & 3,51559 & $1,87411 \times 10^{-7}$ & $2,72813 \times 10^{-14}$ & $1,60032 \times 10^{-18}$ \\
& 2 & 4,99943 & 2,55791 & $1,37085 \times 10^{-7}$ & $3,45837 \times 10^{-14}$ & $8,38927 \times 10^{-19}$ \\
\hline
\end{tabular}

Assim como nos resultados do problema-modelo 1, neste caso-exemplo, o MSD apresentou resultados com boa precisão, quando comparados com a referência de malha fina (DD). Além disso, obteve o mesmo resultado que o método SDM, dentro das casas decimais de precisão avaliadas aqui, uma vez que ambos os métodos de malha grossa usam a solução analítica geral intranodal da equação de transporte, gerando resultados livres de erro de truncamento. 


\subsection{Problema-modelo 3}

No terceiro problema-modelo apresentando neste trabalho, é considerado um domínio heterogêneo composto por duas zonas materiais, considerando espalhamento linearmente anisotrópico. Na Fig. 7]são mostrados os parâmetros físico-materiais que compõem as 3 regiões do modelo, bem como suas espessuras e as condições de contorno.

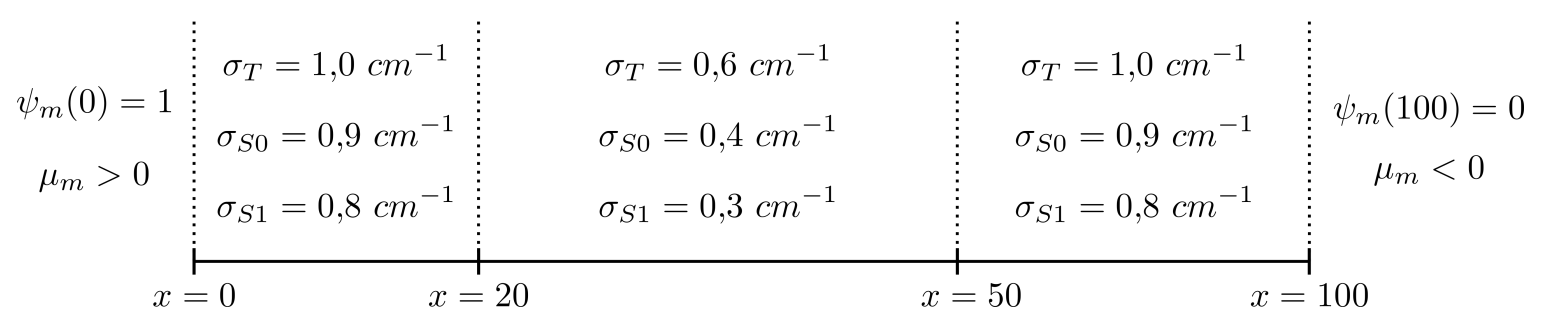

Figura 7: Problema-modelo 3

Na solução deste problema, foi considerado um parâmetro de tolerância $\xi=10^{-6}$. Nos métodos de malha grossa, foi considerado um nodo por região. Para o método DD, foram usados 6000 nodos na primeira região, 15000 na segunda e 9000 na terceira. Os resultados obtidos para os fluxos escalares de nêutrons calculados usando a ordem de quadratura $N=512$ são mostrados na Tabela 4

Tabela 4: Fluxos escalares de nêutrons $\left(\mathrm{cm}^{-2} \mathrm{~s}^{-1}\right)$ - problema-modelo 3.

\begin{tabular}{lcccc}
\hline & \multicolumn{4}{c}{$N=512$} \\
\cline { 2 - 5 } Método & $x=0 \mathrm{~cm}$ & $x=20 \mathrm{~cm}$ & $x=70 \mathrm{~cm}$ & $x=100 \mathrm{~cm}$ \\
\hline DD & $6,11930 \times 10^{-1}$ & $4,09121 \times 10^{-3}$ & $3,22760 \times 10^{-11}$ & $2,42033 \times 10^{-14}$ \\
MSD & $6,11930 \times 10^{-1}$ & $4,09121 \times 10^{-3}$ & $3,22761 \times 10^{-11}$ & $2,42036 \times 10^{-14}$ \\
SDM & $6,11930 \times 10^{-1}$ & $4,09121 \times 10^{-3}$ & $3,22761 \times 10^{-11}$ & $2,42036 \times 10^{-14}$ \\
\hline
\end{tabular}

Ao analisar os resultados dos fluxos angulares de nêutrons deste problema-modelo, pode-se constatar que o MSD apresentou resultados precisos quando comparados tanto com o SDM quando com o método de referência de malha fina DD.

\section{Conclusões e propostas futuras}

Neste trabalho, foram mostrados resultados numéricos de um método espectronodal (malha grossa) aplicado na solução de problemas de blindagem de nêutrons em geometria unidimensional, na formulação de ordenadas discretas, considerando a teoria de multigrupos de energia. O método nodal apresentado, denominado MSD, utiliza a solução geral local intranodal da equação de transporte de nêutrons $S_{N}$, e um processo iterativo que utiliza a equação de balanço espacial $S_{N}$ para calcular os fluxos angulares de nêutrons nos nodos que compõem o domínio estudado. Foram executados 3 problemas-modelo e comparada a precisão dos resultados numéricos dos fluxos escalares de nêutrons gerados pelo MSD com o SDM e o tradicional método de malha fina DD, usado como referência da validação destes resultados.

A análise dos resultados numéricos obtidos nos problemas-modelo apresentados no trabalho, indicam que o MSD foi capaz de gerar resultados com boa precisão, quando comparado com o método de referência (DD) dentro das condições testadas. Além disso, o MSD obteve os mesmos resultados do método SDM, visto que ambos os métodos são da classe dos espectronodais, e que para problemas unidimensionais, geram resultados numéricos sem erro de truncamento. Sendo assim, pode-se concluir que o método MSD foi capaz de resolver os problemas-modelo estudados neste trabalho, evitando o uso de operações matriciais no cálculo dos fluxos angulares de nêutrons emergentes nas interfaces nodais.

Futuramente, pretendemos aplicar o método MSD na solução de problemas multidimensionais, com maior número de grupos de energia, grau de anisotropia arbitrário e o estudo de estratégias de paralelização dos algoritmos implementados. 


\section{Referências}

[1] J. J. Duderstadt, L. J. Hamilton et al., Nuclear reactor analysis, $1^{\text {a }}$ ed. New York, USA: Wiley New York, 1976. Disponível em: https://www.wiley.com/en-us/Nuclear+Reactor+Analysis-p-9780471223634

[2] E. E. Lewis e W. F. Miller, Computational methods of neutron transport, $1^{\mathrm{a}}$ ed. New York, USA: Wiley New York, 1993.

[3] O. P. da Silva, "Um método de matriz resposta para cálculos de transporte multigrupos de energia na formulação de ordenadas discretas em meios não-multiplicativos," Tese de doutorado, Programa de Pós-Graduação em Modelagem Computacional, Universidade do Estado do Rio de Janeiro, Nova Friburgo, Brasil, 2018. Disponível em: https://www.bdtd.uerj.br:8443/handle/1/13795

[4] R. B. Libotte, "Método de malha grossa para solução numérica de problemas de blindagem de nêutrons em geometria unidimensional na formulação de ordenadas discretas com perspectivas a cálculos multidimensionais em geometria retangular," Dissertação de mestrado, Programa de Pós-Graduação em Modelagem Computacional, Universidade do Estado do Rio de Janeiro, Nova Friburgo, Brasil, 2021. Disponível em: https://www.bdtd.uerj.br:8443/handle/1/16461

[5] A. M. Oliva, "Método espectral determinístico para a solução de problemas de transporte de nêutrons usando a formulação das ordenadas discretas," Tese de doutorado, Programa de Pós-Graduação em Modelagem Computacional, Universidade do Estado do Rio de Janeiro, Nova Friburgo, Brasil, 2018. Disponível em: https://www.bdtd.uerj.br:8443/handle/1/13719

[6] R. C. de Barros e E. W. larsen, "A spectral nodal method for one-group x, y-geometry discrete ordinates problems," Nuclear Science and Engineering, vol. 111, no. 1, pp. 34-45, 1992. Disponível em: https://doi.org/10.13182/NSE92-A23921

[7] R. C. de Barros, "A spectral nodal method for the solution of discrete ordinates problems in one- and two-dimensional cartesian geometry," Tese de doutorado, University of Michigan, UMICH, Ann Arbor, USA, 1990. Disponível em: https://deepblue.lib.umich.edu/handle/2027.42/105093

[8] L. B. Barichello, L. C. Cabrera, e J. F. Prolo Filho, "An analytical approach for a nodal scheme of two-dimensional neutron transport problems," Annals of Nuclear Energy, vol. 38, no. 6, pp. 1310-1317, 2011. Disponível em: https://doi.org/10.1016/j.anucene.2011.02.0042

[9] G. I. Bell e S. Glasstone, Nuclear Reactor Theory, $2^{\mathrm{a}}$ ed. Washington, DC: Krieger Pub Co., 1970.

[10] L. B. Barichello, R. D. M. Garcia, e C. E. Siewert, "A spherical harmonics solution for radiative-transfer problems with reecting boundaries and internal sources," Journal of Quantitative Spectroscopy and Radiative Transfer, vol. 60, pp. 247-260, 1998. Disponível em: http://repositorio.ipen.br/handle/123456789/7212

[11] L. B. Barichello e C. E. Siewert, "A discrete ordinates solution for a non-grey model with complete frequency redistribution," Journal of Quantitative Spectroscopy and Radiative Transfer, vol. 62, pp. 665-675, 1999. Disponível em: https://doi.org/10.1016/S0022-4073(98)00096-X

[12] M. Benassi, R. M. Cotta, e C. E. Siewert, "The $p_{n}$ method for radiative transfer problems with reflective boundary conditions," Journal of Quantitative Spectroscopy and Radiative Transfer, vol. 30, pp. 547-553, 1983. Disponível em: https://doi.org/10.1016/0022-4073(83)90010-9 